\title{
Towards realistic modeling of plasmonic nanostructures: a comparative study to determine the impact of optical effects on solar cell improvement
}

\author{
Gholamhosain Haidari ${ }^{1,2}$ (1) \\ Received: 28 April 2021 / Accepted: 29 November 2021 / Published online: 20 January 2022 \\ (c) The Author(s), under exclusive licence to Springer Science+Business Media, LLC, part of Springer Nature 2021
}

\begin{abstract}
Plasmonic structures may improve cell performance in a variety of ways. More accurate determining of the optical influence, unlike ideal simulations, requires modeling closer to experimental cases. In this modeling and simulation, irregular nanostructures were chosen and divided into three groups and some modes. For each mode, different sizes of nanoparticles were randomly selected, which could result in pre-determined average particle size and standard deviation. By $3 \mathrm{D}$ finitedifference time-domain (3D-FDTD), the optical plasmonic properties of that mode in a solar cell structure were investigated when the nanostructure was added to the buffer/active layer of the organic solar cell. The far- and near-field results were used to compare the plasmonic behavior, relying on the material and geometry. By detailed simulations, $\mathrm{Al}$ and $\mathrm{Ag}$ nanostructure at the interface of the $\mathrm{ZnO} /$ active layer can improve organic solar cell performance optically, especially by the near-field effect. Unlike Au and relative Ag, the Al nanostructured sample showed less parasitic absorption loss.
\end{abstract}

Keywords Plasmonic nanostructure $\cdot$ Realistic modeling $\cdot$ Parasitic absorption loss $\cdot$ Near-far field effect $\cdot$ Organic solar cell

\section{Introduction}

Crises may always arise in human society and pose serious emergencies to us. One example is the spread of the COVID-19 virus in 2020. Such cases remind us that restrictions must always be considered before they become serious concerns. Today, researchers warn of current limitations on energy, environmental, and related economic matters that could lead to serious issues in the future [1]. Interestingly, solar energy has sufficient potential to prevent such problems $[1,2]$. Research on photovoltaics has led to the offering of several different types of solar cells [3]. In photovoltaics, increasing efficiency, lifetime, and reducing costs have continuously been considered. The organic solar cell (OSC) has emerged as a leader in internal photovoltaic competition [4].

Gholamhosain Haidari

haidari@sku.ac.ir; moh1135@gmail.com

1 Department of Physics, Faculty of Sciences, Shahrekord University, Shahrekord, Iran

2 Nanotechnology Research Institute, Shahrekord University, Shahrekord, Iran
The silicon-based cells usually involve high cost of materials and their processing. Moreover, perovskite cells have shown a significant increase in efficiency, but they have not shown good stability so far. Therefore, organic cells are still considered a viable alternative for first-generation cells [5, 6]. It is possible to produce roll-to-roll and economic and lightweight organic cells with flexibility and proper efficiency and stability $[3,4]$. However, OSC efficiency is relatively low compared to other competitors. Low carrier mobility in most organic semiconductors limits the thickness of the active layer, decreasing light absorption [4]. Therefore, increasing the light absorption of organic semiconductors has become a challenge due to this limitation. In 1998, Stuart and Hall observed that by introducing silver nanoparticles (Ag NPs) on the surface of a silicon photodetector, a nearly 20 -fold enhancement in photocurrent was obtained. The results indicated that metallic NPs could induce local surface plasmon resonance among themselves or scatter light [7]. This outcome is significant in light-harvesting applications such as water splitting, photodynamic therapy, thermoelectric, photovoltaic, and photocatalytic technology [8]. In photovoltaics, such as OCS, the metal nanostructures (MNSs) may act as a light-trapping mechanism [9-11]. They 
can enhance the absorption in the active layer while the thickness is still very thin (around $100 \mathrm{~nm}$ ). In fact, concerning plasmonic NSs in solar cell technology (especially in the active layer) apart from light trapping, two other issues have also been considered: (1) detailed balance limit, (2) concept of optical metamaterial (or effective medium theory).

Fundamental thermodynamic limits for idealized solar cell devices with maximum efficiencies and optimum light trapping are given by the Shockley-Queisser (SQ) limit and the Yablonovitch (or Lambertian) limit. The optical properties of the device and any concepts for light trapping are not considered in the original SQ theory [12]. Moreover, for the conventional light-trapping system such as roughening the semiconductor-air interface, the standard theory can show that the absorption enhancement factor has an upper limit of $4 n^{2}$ at normal incidence (Yablonovitch limit), where $\mathrm{n}$ is the refractive index of the absorbing layer [13, 14]. But it has been shown that in a nanophotonic regime such as the plasmonic NS with the proper design, the absorption enhancement factor can go far beyond this limit. For instance, it was demonstrated that the enhancement factor can, in theory, be increased to $12 \times 4 n^{2}$ over a virtually unlimited spectral bandwidth when optical modes exhibit deep subwavelengthscale field confinement [15].

Despite recent proper developments in perovskite cells, its efficiency is still low than the SQ limit [16]. Therefore, the metamaterial approach of plasmonic structures can also be helpful in this regard [16]. Prior reports on embedding plasmonic NPs into active layers focused mainly on nearfield enhancement by localized surface plasmon resonance (LSPR) or antenna effect. But LSPRs of plasmonic NPs and their capacitive coupling increases not only near-field absorption but also as a plasmonic metamaterial cause change in the effective refractive index $\left(n_{\text {eff }}\right)$ of the medium [17-19]. It was shown that the key point for increasing the $n_{\text {eff }}$ (particularly beyond the solar cell bandgap), is to maximize the effective permittivity $(\varepsilon)$ and to suppress effective permeability $(\mu)[16]$. The dipolar interaction between MNPs can affect the polarization and associated effective permittivity, whereas the diamagnetic effect can be precisely controlled by adjusting the ratio of skin depth $(\delta)$ to the thickness $(d)$ of NP. According to this reference [16], as the density of photo-generated carriers defines voltage, boosting photon-recycling by increasing $n_{\text {eff }}$ results in a reduction in $V_{\mathrm{oc}}$. Moreover, it was concluded that $V_{\mathrm{oc}}$ and $J_{\text {sc }}$ trade-off with each other, once both light trapping and photon-recycling are simultaneously boosted by increasing $n_{\text {eff }}$ Therefore, this suggests that plasmonic metamaterials are effective only for ultrathin perovskite solar cells (thinner than $100 \mathrm{~nm}$ ). The enhancement of light absorption via increasing refractive index, particularly beyond the band gap of the active layer, has been less reported for OSC. Moreover, in the case of photon recycling, photons may undergo multiple reabsorption and re-emission events within the absorber. They may bounce back and for many times within a thin-film solar cell, the optical design and trap passivation (Shockley-Read-Hall and Auger recombination) must play a vital role in limits of power conversion efficiency [20].

MNSs with various sizes and morphologies have been generally used in photovoltaics to activate plasmonic consequences [21]. Depending on their position inside OPV, it usually leads to three conventional optical mechanisms, namely (1) light scattering, (2) LSPR, (3) surface plasmon polaritons (SPPs). However, MNPs can be the origin of nonoptical effects inside the cell. They can have other positive or negative effects on solar cell (SC) performance, such as electrical effects (such as plasmon-induced charge separation, change in surface resistance, change in electron-hole transition, change in surface recombination), and changing layer morphology $[9,11,21]$. Therefore, by considering the various effects mentioned above, improving the performance of the OSCs through metallic NSs can be a complex mechanism. For the OSC, the MNSs have been inserted into relatively distinct positions: into the buffer or active layer, at the bottom interface between the active layer and the metal electrode, or the interface of two layers. If the plasmonic enhancement more focuses on further scattering of the incident light into the active layer, the MNSs can be placed on (1) the interface of the transparent conductive layer (TCO) and carrier transport layer (CTL), (2) directly inside CTL or (3) the CTL-active layer interface.

Concerning the material selected for this purpose, most $\mathrm{Ag}$ and $\mathrm{Au}$ NSs have been used to date. Both metals have good chemical stability and have been studied in various methods to make regular and irregular NSs [22]. But for these noble metals, plasmonic wavelength (and most likely parasitic absorption) is usually in the visible region, where it often overlaps with the absorption region of the active layer.

Parasitic absorption is another primary practical limit, which refers to the loss of photocarriers excited in the metal particles themselves that decay via nonradiative channels to produce heat. By controlling the size, morphology, material, and placement of the metallic NPs, parasitic absorption can be minimized.

As plasmonic centers, the plasmonic resonance modes of an NP can result in either forward-backward scattering or parasitic absorption. Aluminum ( $\mathrm{Al})$ has emerged as an alternative metal due to its low cost and LSPR in the UV spectral region. For example, according to Mie's theory, for a sphere with a diameter of $60 \mathrm{~nm}$ of $\mathrm{Ag}, \mathrm{Au}$, and $\mathrm{Al}$ in the air, extinction peaks occur at $380 \mathrm{~nm}, 504 \mathrm{~nm}$, and $208 \mathrm{~nm}$, respectively. Al is a nonresonant plasmonic metal, which means that the LSPR of Al may be more easily engineered. Therefore, it cannot interact with the more significant amount of incident light (i.e., visible spectrum), and consequently, plasmon-induced losses by parasitic absorption 
does not occur. In 2010, the effects of resonant (Ag) and non-resonant $(\mathrm{Al})$ plasmonic NPs in a thin-film hydrogenated amorphous silicon (a-Si:H) solar cell were studied [23]. In 2014, the enhancing of the optical absorption of the dyesensitized solar cell (DSC) by Al NS was investigated [24].

In 2014 and 2015, the finite-difference time-domain (FDTD) analysis of solar absorption enhancement in perovskite films embedding plasmonic nanoparticles was studied. The plasmonic near-field enhancement and scattering effects as a function of different shapes, size, composition, concentration, dimer formation, and to some extent, random state were analyzed [25, 26].

For preparing MNSs, depending on the desired size and geometry, many physical and chemical fabrication methods have been used [27]. Each of these methods has its advantages and disadvantages. For example, the thermal evaporation method is a simple, clean and environmentally friendly method for preparing NSs [27]. The potential plasmonic benefits of Al-NS over Ag and Au depend on its plasmonic wavelengths. But, that is a function of the size, shape, and surrounding material [21]. Therefore, determining the plasmonic wavelengths and usability of MNS in an SC is of great importance.

In this study, using the 3D-FDTD simulation method, the plasmonic effect of $\mathrm{Al}$ for improving the performance of organic cells was investigated. In this study, MNS modeling was performed to suitably represent somewhat regular NSs (in the TCO/CTL and CTL/active layer interface) formed by some experimental methods. Therefore, this MNS model was designed to be closer to experimental models. To better clarify the plasmonic effect, the near- and far-field simulated results of $\mathrm{Ag}$ and Au NSs (with a similar structure) were compared with Al.

Beyond evaluating the absorption enhancement factor, Schuster et al. proposed a figure of merit called lighttrapping efficiency (LTE), the ratio of the total current gain achieved in a device to the theoretical maximum current gain achievable in an ideal Lambertian scattering system. Using LTE as a metric, they showed that multiple theoretical and experimental photonic structures could be used to approach the enhancement limit [28]. In this case, it was again emphasized that plasmonic enhancement strategies ideally fall under the class of approaches that can be used to exceed the Lambertian limit. They stated that the performance of theoretical structures is significantly superior to that of experimental ones. This points to the need for theoretical studies to better take "real" effects such as parasitic absorption into account. Therefore, in this modeling, special attention was paid to parasitic absorption. They also point out that achieving experimental ideal light-trapping structures is more complicated than designing them theoretically.

Moreover, the outcome of this research could help in determining the optical effect of $\mathrm{Al}, \mathrm{Ag}$, and Au NSs in a variety of solar cells, such as dye-sensitized and perovskite solar cells.

\section{Theory and modeling}

\subsection{Aluminum against noble metals}

Noble MNSs such as Au and Ag have been widely used in plasmonics due to their relative ease of production. In contrast to noble metals, $\mathrm{Al}$ is low-cost and generally available, as it is abundant on Earth. Noble metals may produce a negative optical effect and reduced performance of the SC in the LSPR wavelengths due to absorption losses. However, they may show a positive impact and photocurrent enhancement in the off-resonance section. The resonant wavelength of $\mathrm{Al}$ is generally located in the UV region but can be extended to the visible or even near-infrared region [29]. Moreover, the use of Al plasmonics has advanced considerably in recent years [30]. Colloidal chemistry of Ag and Au, based on salt reduction, is broadly employed in various applications in nano-optics. In contrast, the chemical synthesis of Al-NP is not a common method, since the reduction of $\mathrm{Al}$ salts is complicated [31]. Simple metal evaporation followed by different thermal annealing and masking techniques can lead to well-separated NSs over large areas [32-34]. Although annealing and using a mask can lead to a relatively regular pattern of NSs, this method generally points to NS with varying distribution in shape and size.

\subsection{A glance at conventional modeling}

In numerical electromagnetics, a method such as finite-difference time-domain (FDTD) is beneficial when a broadband analysis is required, such as in the SC. The FDTD method uses the central difference approximation to discretize the Maxwell equations, in both the time and spatial regions. It then derives the electric and magnetic field distributions in the Yee cells at each time step [35, 36]. In a conventional SC FDTD simulation, the 3D simulation cell includes different thin-film cell layers. The plane wave source is polarized along with thin films and propagates in the depth direction. Therefore, the periodic boundary conditions (PBC) are set in line with the thin layers. The perfectly matched layer (PML) boundary condition is selected perpendicular to them. The PML is an advantage of the FDTD method compared to the finite element method (FEM), making it possible to more appropriately simulate problems that have unclosed space [36]. Different dispersive materials are identified in the simulation cell due to their complex refractive indexes. Moreover, it is possible to calculate the reflection-transition spectra and parasitic absorption of different layers by the simulation. In this conventional simulation (i.e., the 1D 
photonic crystal), this type of calculation can also be done to some extent with a more straightforward method such as the transfer matrix method (TMM). FDTD capabilities manifest when the optical model tries to contain more detail. Simulation of plasmonic SC is one case where the FDTD method has proven its ability to simulate the near and farfield and SPP effects [35]. Plasmonic SC mainly refers to the use of plasmonic NS to overcome the limitations of thirdgeneration SCs such as OSC and perovskite cells [21, 37]. Initially, more attention was paid to the light trapping due to the plasmonic NSs. But they can be involved in improving the performance of OSC through various optical, electrical, and structural mechanisms [10], and optical effects can be considered one of the most important. There have been some simulations about the optical study of MNSs concerning SCs. These MNs can be added at different SC locations, and depending on the location, the near- and far-field effect or both on the efficiency of the SC can be examined [9-11]. In the far-field approach, the reduction in backward scattering is generally simulated, leading to an increase in the optical absorption of the active layer. In the near-field process, the determination of wavelength(s) of LSPR is the simulation's goal. In typically simulated structures, they mainly consist of structures composed of a single particle (such as a sphere or hemisphere) and sometimes periodic nanostructures (NSs) [38]. Concerning a single particle, the simulations can only be practical regarding the particle distribution with a considerable distance (single-particle approximation). However, if more attention is paid, the experimental samples do not have NPs with the same shape. Therefore, it cannot be expected that the near-field distribution around all of them will be the same, limiting the applicability of the results of such simulations. Moreover, about periodic structures, the results of far-field simulations depend on the shape and size of the NS and the periodic specifications. Again, in an experimental sample, one cannot expect a periodic structure to be precisely the same as the optimized numerical structure. On the other hand, the results of such simulations show more weaknesses, especially when it comes to simulating accurately experimental periodic NSs. In fact, in the often used method of NS fabrication, the shape, size, and periodicity distribution of the MNS will be, to some extent, wide. Therefore, the more accurate FDTD modeling of these nanostructures will have its challenges.

To address this challenge in this study, due to the experimental data concerning experimental MNSs [23, 29, 30, $32,33,39,40]$, firstly, elliptical nanoparticles were chosen and divided into three groups based on the oblate or prolate form. Moreover, by vertical height, some modes were considered for each group. For each mode, different sizes of nanoparticles were somewhat randomly selected, which could result in pre-determined average particle size and standard deviation. They were put on the interface of the
ITO/buffer layer and buffer/active layer, respectively. In this study, for buffer/active case, the viewpoint of increasing the $n_{\text {eff }}$ was not considered. This type of modeling can represent, to some extent, orderly MNSs that can be formed by some experimental methods.

\subsection{Details of this modeling}

In the fabrication of nanostructure, the nucleation mechanism of thin-film growth has been classified into three major types (Frank-Van der Merwe or island growth, Volmer-Weber or layer growth, and Stranski-Krastanov) depending on the interaction between the deposited atoms of the target and the surface of the substrate [41]. The metal forms the NS shape since the thickness $(<\sim 30 \mathrm{~nm})$ is not thick enough to create a continuous film [32]. Atoms undergo a series of kinetic processes, then initiate nucleation and island formation. The nanostructure fabrication method can be associated with various conditions, such as heating the substrate during formation and annealing. For example, thermal evaporation by using different masks leads to more structured arrangements and separate NPs. In some fabrication methods depending on the various conditions, particles can form a shape similar to an ellipsoid (or part of it) and with different contact angles. On the other hand, the review of atomic force microscopy (AFM) and scanning electron microscopy (SEM) images in some literature suggests that the ellipsoidal NPs for modeling experimental NS is a suitable choice $[23,29,30,32,33,39,40,42,43]$. These ellipsoidal NPs are to some extent randomly distributed in shape, size, and distance from each other. Usually, average values of these geometrical factors are used for modeling, i.e., the distribution of identical NPs with an average size at a constant distance (average distance) from each other [43]. Since the plasmonic properties are very dependent on the shape, size, and distance distribution of the NPs, the conventional methods cannot be effective for the simulation. Therefore, in this study, an attempt has been made for more accurate modeling of these kinds of MNSs. Consider NPs by combining ellipsoids with the random distribution (i.e., to some extent, random ellipsoids in shape, size, rotation around vertical axes, and distance). In this modeling, the NPs were divided into three groups: oblate ellipsoid, prolate ellipsoid, and oblate spheroid (O, P, and S). For each group, some modes (specified with a number such as $1,2,3$, and 4) were considered, as shown in Fig. 1. These three groups and their modes are somewhat reminiscent of different states (different particle heights, elongation, or oblation and different contact angles) deposited on the substrate by the various experimental methods. Therefore, the simulation of these states will be beneficial to predict the near-far optical field effects. Mean particle sizes with their standard deviation supposed for each mode are listed in Fig. 1. Then, assuming 
Oblate-like ellipsoidal NS

\begin{tabular}{|c|c|c|c|}
\hline 4 Modes & a & b & c \\
\hline Average (nm) & 47 & 58 & 12 \\
\hline $\begin{array}{c}\text { Standard } \\
\text { deviation (nm) }\end{array}$ & 5 & 11 & 6 \\
\hline
\end{tabular}

$\frac{4}{4} C$

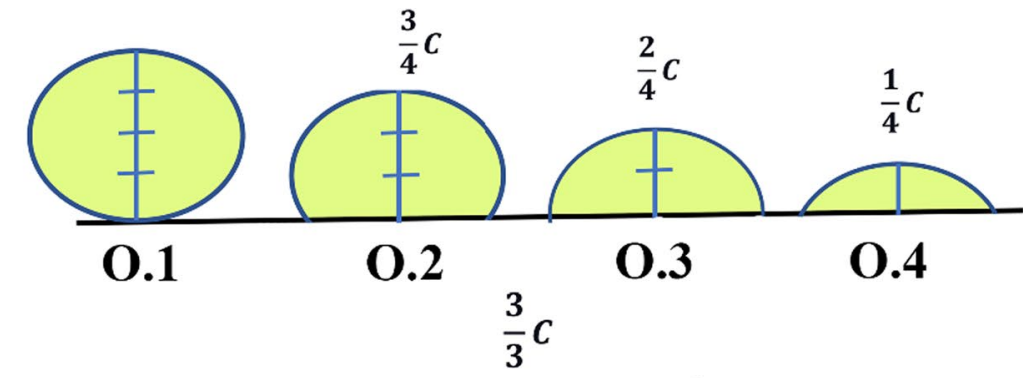

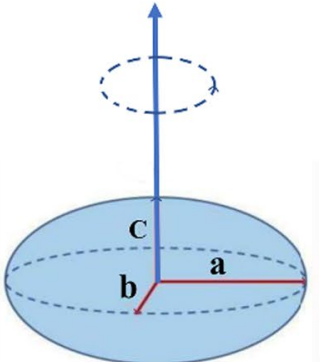

Material: Al or Ag or Au

\section{Oblate-like Spheroidal NS}

\begin{tabular}{|c|c|c|c|}
\hline 4 Modes & a & b & c \\
\hline Average (nm) & 47 & 47 & 12 \\
\hline $\begin{array}{c}\text { Standard } \\
\text { deviation (nm) }\end{array}$ & 5 & 5 & 6 \\
\hline
\end{tabular}

Fig. 1 Demonstration of the three groups, O, P, and S. They refer to oblate, prolate and spherical particles, respectively. The geometric characteristics of each group (pre-determined average particle size and standard deviation) are mentioned in the tables. Each group has

the average particle sizes and their standard deviations for each mode, some nanoparticles with different geometrical parameters were chosen for making MNS. The steps of the modeling algorithm are given in the Supporting Information.

Figure 2 shows the 3D perspectives and $\mathrm{XY}-\mathrm{XZ}$ view of a typical MNS of one mode. This figure also shows the cross-section planes perpendicular to the $\mathrm{XZ}$ surface (normal cross-section).

In addition, quantum effects influence the plasmonic effect of smaller NPs [44], although they seem more relevant for a size smaller than $\sim 2 \mathrm{~nm}$ [45]. This happens because of the optical constant modification when approaching the quantum regime, known as 'non-local effects'. The standard description of optical properties of metal NPs exploits a 'local' bulk dielectric function, i.e. $\varepsilon(\omega)$ only depends on frequency but not on NP size, shape, and structure [46]. However, this approach fails for very small diameters [44-46]. Non-locality also has relevant implications when small metal NPs are close to each other (the gap varying from -19 to $+19 \AA$ ) [47]. modes that differ only in the vertical particle size (here ' $c$ ' axis). Simulations are done separately for each mode and material (Al, Ag, and $\mathrm{Au})$

Quantum effects were not included in this FDTD simulation. Some studies have been done to include these missing effects, for example, by using the hydrodynamic Drude model in the conventional FDTD method [48-50] or nonlocal classical theory employing the transformation-optics approach [51, 52]. In this study, as much as possible, while the simulated structures were close to some experimental studies, quantum details were not considered. Therefore, the particles were distributed so that the distances between the particles were not less than $20 \mathrm{~nm}$ [45, 53]. Moreover, while the possibility of particles entering together to create more irregular particles had been considered, this was done in such a way that the gaps between NPs were less than or equal to $-5 \mathrm{~nm}[47,54]$. In each random particle distribution for meeting the requirement (such as the minimum distance between particles), the shape of the MNS was qualitatively checked before simulation with software such as ImageJ. Otherwise, by changing the random number in the programmed script, another distribution of particles was checked. 

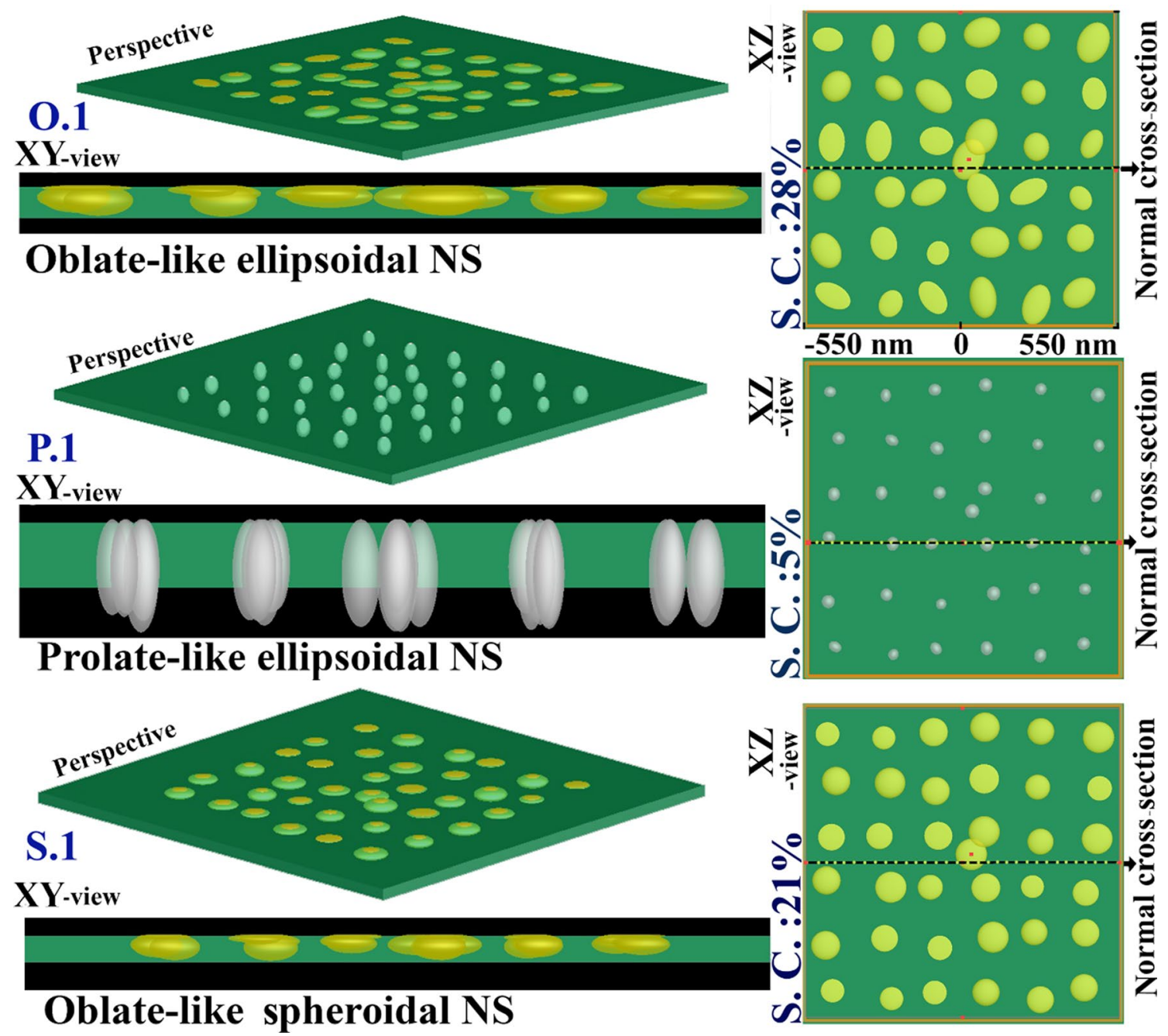

Fig. 2 Perspectives and $X Y-X Z$ view of the model for the three groups $(\mathrm{O}, \mathrm{P}$, and $\mathrm{S})$. The $X Z$-view shows the surface on which the particles have the largest lateral diameter. The distance of the center of the particles from each other on all surfaces is the same and its average size is $140 \mathrm{~nm}$. In this case, the percentage of surface cover-

\subsection{Simulation features}

Figure 3 shows the 3D-FDTD model of OSC:ITO/ZnO/ MNS/PTB7-PCBM/MoO3/Al. The plane-wave source and the FDTD region with the perfectly matched layer (PML) in the y-direction and Bloch boundary condition $(\mathrm{BC})$ in the $\mathrm{XZ}$ surface are shown. The Bloch $\mathrm{BC}$ is very similar to the periodic $\mathrm{BC}$, but because of the random distribution of MNS, while copying the fields from one edge to the other, they also apply a phase correction to the fields. The refractive index and extinction coefficient as a function of wavelength of different materials for the simulation were taken from reliable sources such as [55]. The sample data option with the multi-coefficient material model of the Lumerical simulation software with the best fit was used to age (S. C.) was also reported. The selected (typical) normal cross sections (including $Y$ - and $Z$-axes) are shown as a dotted line. In $O .1$ and S.1, they contain only a part of one particle of MNS. However, P.1 includes several particles

introduce new material into the simulation. In the model, the flux monitors are located on the top outside of the structure to measure reflected light (backward scattering) at various wavelengths. For far-field such as total reflection, the results were presented after averaging the data of ten simulations. In these ten simulations for each mode, the random distribution of particles was different, but the mean values determining the distribution characteristic of metallic NS remained identical. The FDTD approach includes responses from a wide range of frequencies that are widely used for numerical simulation of the optical properties of the plasmonic NS with a single run. The Lumerical simulator is used for FDTD simulation [56]. To better consider the NS in simulation, the override mesh setting $\left(2 \times 2 \times 2 \mathrm{~nm}^{3}\right.$ Yee cell $)$ was applied to this section of the OSC model. A cross-section surface with 
Fig. 3 Illustration of 3D-FDTD region of the simulation. In the inset, three normal cross sections are shown; they are also shown as the dotted lines in Fig. 2. In the simulation, nearfield results are calculated on these slices

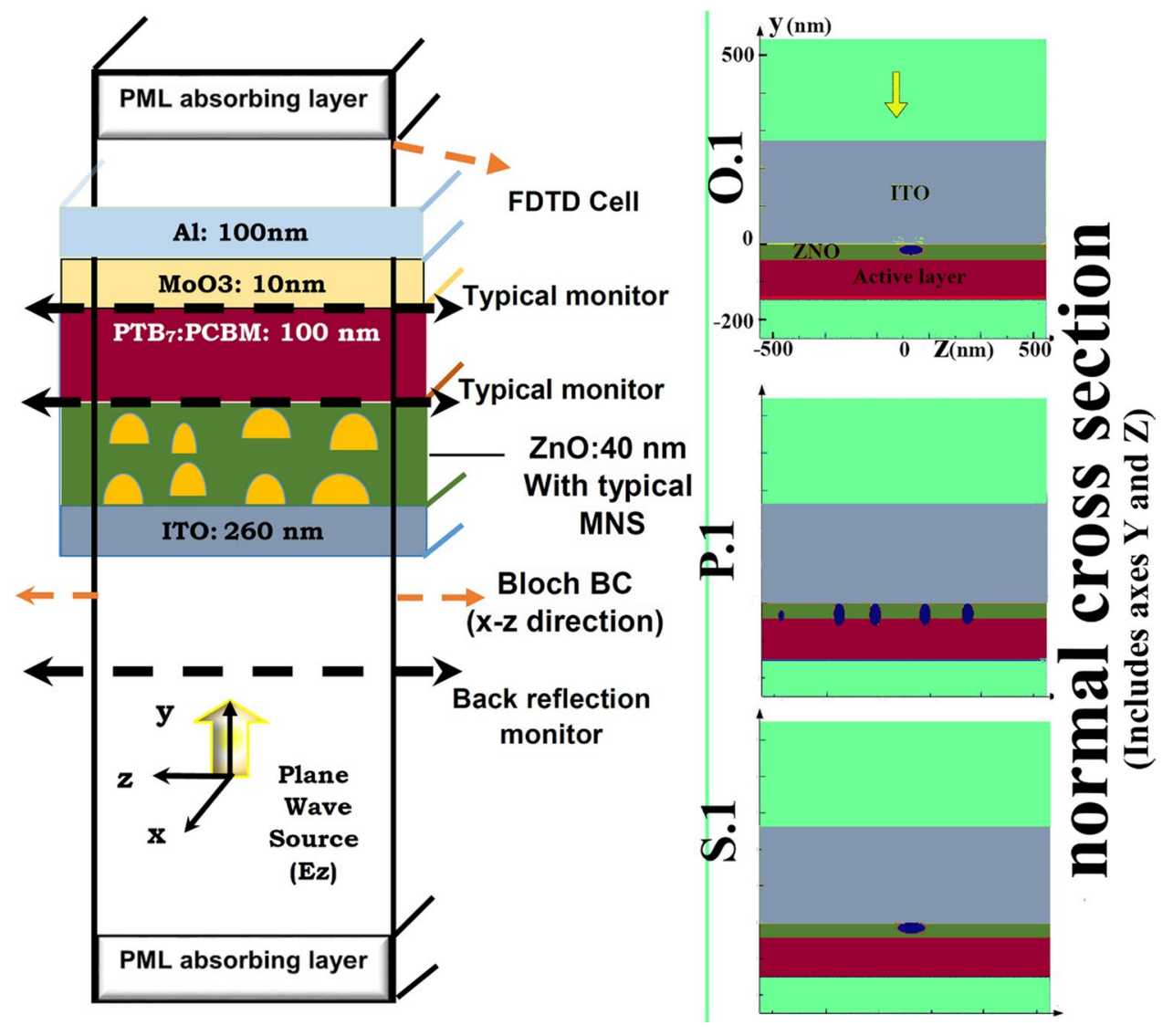

the $y-z$ axes was also selected for routine near-field monitoring (Fig. 3). On the other hand, the maximum field intensity $\left(E_{\max }^{2}\right)$ on this surface was recorded at each wavelength. The random distribution of particles in terms of number, size, and distance depends on the selected cross section. Thus, the geometry of the NS and, therefore, the near-field on each plane can be different. In other words, the near-field intensity will vary from one surface to another surface. However, the calculated $E_{\text {max }}^{2}$ on this selected surface can compare the near-field effect when the material of particles changed among $\mathrm{Ag}, \mathrm{Au}$, and $\mathrm{Al}$.

\section{Result and discussion}

\subsection{Planar standard solar cell}

Since the FDTD is a space and time domain technique, the fields are calculated as a function of time across some spatial regions within the simulation domain. Then, the Fourier transform gives the fields as a function of frequency (spectral calculation). By propagating power across the surface, the normalized transmission reflection can be calculated. Figure 4 shows the total reflection curve of the standard SC structure introduced in Fig. 3 (without the presence of the

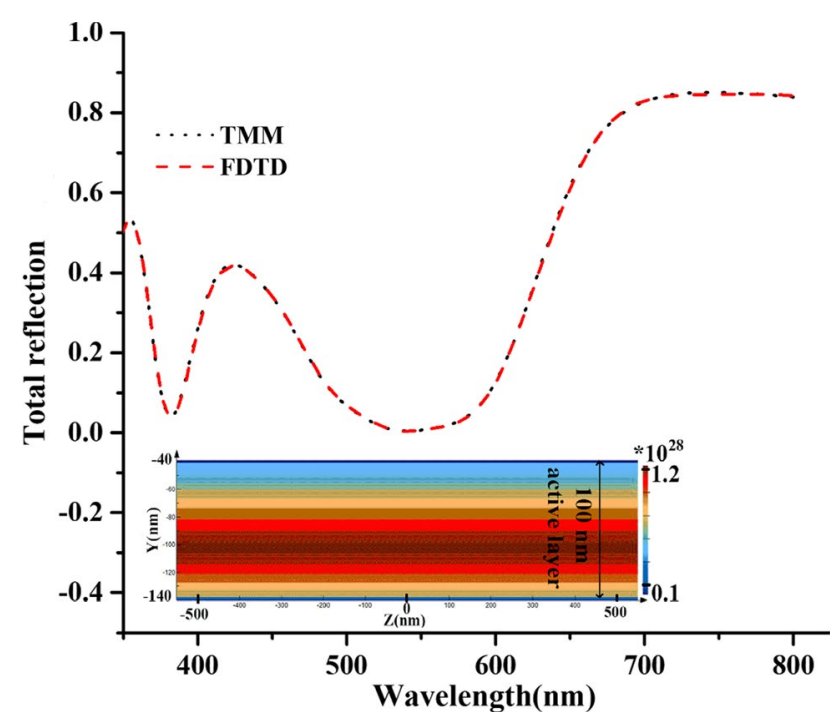

Fig. 4 The total spectral reflection (backward scattering) of the standard cell was calculated by TMM and FDTD methods. The inset shows the generation rate profile of the standard cell

MNS). This curve, because of the coherence interference, has peaks and valleys and can be engineered according to changing the thickness of layers and dispersive reflective indexes. 
For a standard SC with a thin-film structure, the correspondence of the FDTD with the TMM method is well seen in Fig. 4. The optical power absorbed per unit volume can also be calculated by the FDTD. The photon absorption rate is equivalent to the generation rate when it is assumed that each absorbed photon excites an electron-hole pair. In the inset of Fig. 4, the generation rate is shown along with the active layer of the standard SC, with the highest rate being around its center. In the standard cell, the ideal $J_{\text {sc }}$ (shortcircuit current) of $14.67 \mathrm{~mA} / \mathrm{cm}^{2}$ was calculated. With the addition of MNS, the assumption of a 1D photonic crystal of the cell (or TMM method) is no longer sufficient, and therefore the FDTD technique was used for other results.

\subsection{Plasmonic NS (MNS) at the interface of ITO/ZnO: far-field}

The far-field results (total reflection) of plasmonic cells of the O-group (different modes, O.1-O.4) are shown in Fig. 5, while the material of the plasmonic structure is $\mathrm{Al}, \mathrm{Ag}$, and
$\mathrm{Au}$, respectively. In this part, the MNS is at the interface of ITO/ZnO. As shown in Fig. 5, none of the O-group of MNS cause a reduction in the reflection across the whole spectrum compared to the standard cell.

In $\mathrm{O} .3$ and $\mathrm{O} .4$, the difference between the reflection curve of plasmonic samples and the standard becomes smaller. In O.1 and O.2, from the wavelength of $660 \mathrm{~nm}$ onwards, a decrease in the reflection curve compared to the standard sample is observed. The most significant drop is for $\mathrm{Au}$, then $\mathrm{Ag}$, and finally $\mathrm{Al}$. This behavior is more or less seen for O.3 and O.4. Only the Al sample had reflections below the standard curve in the wavelength range between $\sim 400$ and $\sim 500 \mathrm{~nm}$. The simulated far-field results for groups $\mathrm{P}$ and $\mathrm{S}$ are shown in Figs. S1 and Fig. S2 (supporting information).

In the P-group, except for mode P.2, there is not much change in the reflection curve compared to the standard. In P.2, a decrease in the reflection curve is observed only in the range of 380-530 $\mathrm{nm}$, especially for Al. The total reflection comparison of the O-group, P-group, and the standard
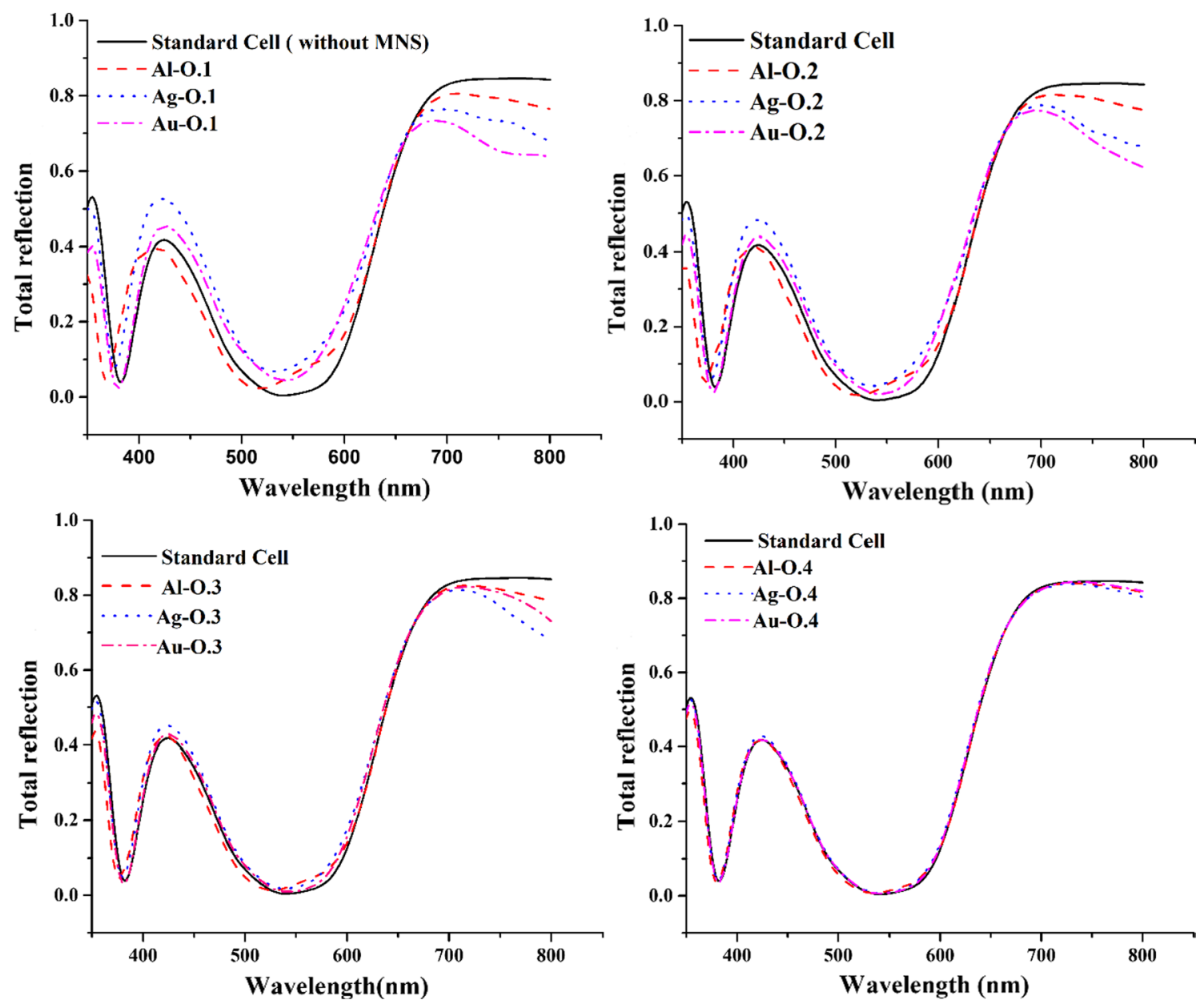

Fig. 5 The total spectral reflection (backward scattering or far-field result) of the plasmonic OSC (MNS at the interface of ITO/ZnO). The MNS is modeled with different O-group modes (O.1 until O.4). The materials used for MNS are $\mathrm{Al}, \mathrm{Ag}$, and $\mathrm{Au}$ 
cell shows the shape impact of MNS on the far-field effect. Comparing Fig. 5 and Fig. S2, there is not much change in the far-field behavior of groups $\mathrm{O}$ and $\mathrm{S}$. The area under the reflection curve in the range of 350-800 $\mathrm{nm}$ can be introduced as a quantity for relative comparison. The normalized value of this quantity to the standard cell $\left(\mathrm{A}_{i} / \mathrm{A}_{s}\right)$ is given in the Supporting Information (Fig. S3). In other words, the lower the $A_{\mathrm{i}} / A_{\mathrm{s}}$, higher the forwarding scattering due to the MNS. It is shown that for Ag, the size of this quantity is more than 1 in groups $\mathrm{O}$ and $\mathrm{S}$ and less than 1 in the $\mathrm{P}$-group. The opposite behavior is observed for $\mathrm{Au}$. For Ag, the rate of change in this quantity from O-S to P-group is more significant. It is seen that for $\mathrm{Al}$, this quantity is less than 1 for all three groups, and its behavior is more uniform than the two others. Therefore, Al may have less dependence on its geometry for reducing the backward reflection.

Moreover, in mode 1, compared to the other modes, the height (in the c direction) of the NP is complete, so they can refer more to nanoparticles prepared using the chemical method. From the viewpoint of contact angle, in mode 2, the contact angle is more than $90^{\circ}$ (little wetting). In mode 3 , the contact angle is $90^{\circ}$, and in mode 4 , the contact angle is less than $90^{\circ}$ (good wetting). Therefore, depending on the ratio of the height of the NP to its lateral surface and material, engineering the contact angle of NSs can be considered one of the factors for the effective scattering of light into the active layer.

\subsection{Plasmonic NS (MNS) at the interface of ITO/ZnO: near-field ( maximum field intensity)}

In the following, the near field of the introduced MNS (groups $\mathrm{O}, \mathrm{P}$, and $\mathrm{S}$ ) was studied. For this purpose, some cross sections were selected. These cross sections are shown in Figs. 2 and 3. On different points of these surfaces, the intensity distribution can be calculated at each wavelength. It is also possible to compare the maximum field intensity $\left(E_{\max }^{2}\right)$ on these cross-sectional surfaces in terms of wavelength. Indeed, the near-field result is due to plasmonic structures and usually occurs near and between them. Because of the somewhat dispersed distribution in the size and position of the nanoparticles, these cross sections show different forms of NPs from one surface to another. Therefore, the near-field results will vary for each one. However, to compare the results of the introduced models of the MNS in the solar cell, such simulated near-field results can be helpful. Figure 6 shows at what wavelength the field intensity is maximized on the selected surface of the O-group with different materials. This near-field effect results from the slice of MNS in the cross section and other adjacent parts of MNS that do not exist on this surface. Al has been recognized as the deep UV plasmonic material, but it can also be tuned as new visible plasmonic material. As can be seen for Al, primarily due to the plasmonic NS in an environment with a higher refractive index than air $\left(n_{\mathrm{ZnO}} \sim 2.4\right)$ in the $\mathrm{SC}$ structure, the peaks of maximum near-field intensity are extended to higher wavelengths than the bulk case. The presence of multiple peaks at spectral maximum intensity can depend on numerous factors in the MNS, such as NP size distribution, NP distances, bipolar resonance, and higher modes such as quadrupole and longitudinal-transverse excitations $[57,58]$. As the height of the nanoparticles decreases in different modes of the O-group (O.1 to O.4), the location of the peaks shift so that the peaks become intensive at larger wavelengths. In Ag-O.1 and Ag-O.2, peaks are seen throughout the visible spectrum. By changing the mode to $\mathrm{Ag}-\mathrm{O} .3$ and Ag-O.4, the number of peaks drops, and the peaks with high intensity will be out of the visible spectrum. The same pattern (more or less) is seen for $\mathrm{Au}$, since the bulk of the plasmonic wavelengths for Au are nearly in the middle of the visible region (unlike $\mathrm{Ag}$, which is at about $400 \mathrm{~nm}$ ). Therefore, Au up to $550 \mathrm{~nm}$ has no significant near-field effect in any of the modes.

For all modes of $\mathrm{Al}$, there are peaks throughout the visible spectrum. In terms of the numerical value of the peaks, higher intensities for $\mathrm{Au}$, then $\mathrm{Ag}$, and finally $\mathrm{Al}$. The spectral maximum field intensity for groups $\mathrm{P}$ and $\mathrm{S}$ are given in Fig. S4 and Fig. S5, respectively. The difference between the O-group and S-group is mainly in their lateral elliptical and circular shape, which causes slight differences in the far-field simulation (Fig. 5 and Fig. S2) but shows significant changes in the near-field effect (Fig. 6 and Fig. S4). The far-field results of various modes of the P-group do not demonstrate much difference in the total reflection spectrum with the standard (Fig. S1). In contrast, Fig. S4 shows that this structure has significant effects in the near-field domain. One of the considerable differences in NS geometry between $\mathrm{O}-, \mathrm{S}-$, and P-group can be considered in the ratio of diameter to height of NPs. The P-group represents more prolate particles and less surface coverage than other groups. As shown in Fig. S4, the lower number of peaks, and to some extent lower widths, indicate that the coupling effects, and generally the effect of adjacent NPs are relatively less in this case. In other words, due to the greater distance among the NPs (or lower surface coverage), this state is closer to the approximation of the independent particle. Therefore, the P-group shows apparent differences between its nearfield effects and other groups. In the P-group (three modes), the field-intensity scale is larger than the other two groups, especially $\mathrm{Au}$. At first glance, this may be attributed to the effects of adjacent particles on each other (the selected cross section for the P-group has more particles than the $\mathrm{O}$ - and $\mathrm{S}$-group). But given that the distance between the particles is considerable, this assumption cannot be significant (the highest intensity was also observed inside the NP). Also, among the three modes of the P-group (Fig. S4), P.2 shows 

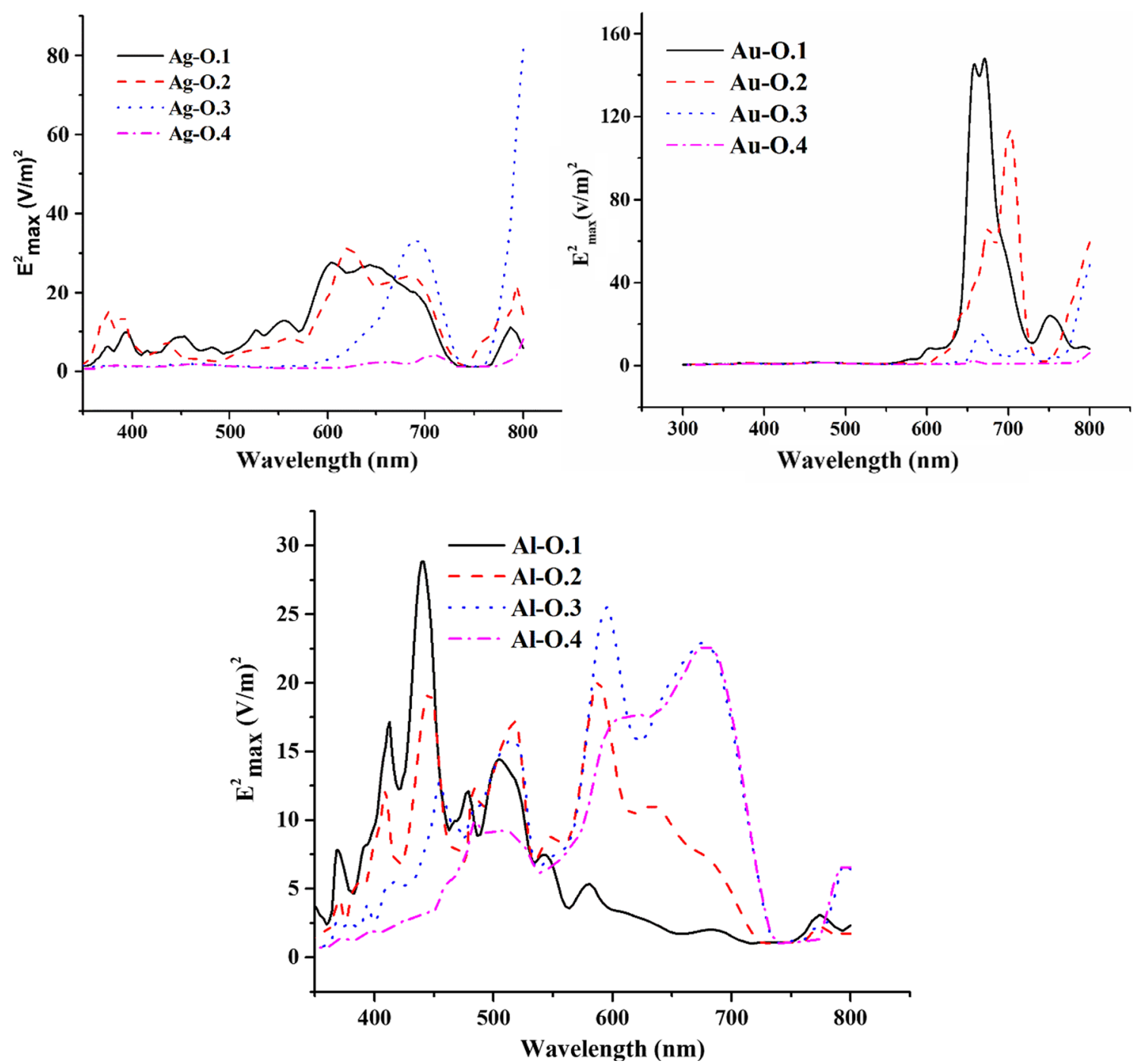

Fig. 6 The spectral maximum field intensity was simulated on the selected cross section for the four modes of O-group and different materials $\mathrm{Al}, \mathrm{Ag}$, and $\mathrm{Au}(\mathrm{MNS}$ at the interface of ITO/ZnO)

a much greater superiority. This highlights the importance of NP geometry.

\subsection{Plasmonic NS (MNS) at the ITO interface/ZnO: near-field (field intensity image)}

In the extension of the near-field study, at the same crosssectional surfaces where the spectral $\mathrm{E}_{\text {max }}^{2}$ was calculated, the electric field intensity $\left(E^{2}(\mathrm{~V} / \mathrm{m})^{2}\right)$ on these surfaces at specific wavelengths $(400 \mathrm{~nm}, 500 \mathrm{~nm}, 600 \mathrm{~nm}, 700 \mathrm{~nm}$, and $800 \mathrm{~nm}$ ) are simulated for some modes. Figures 7 and 8 show these intensity profiles for O.2 and P. 2 modes for Al material, respectively. The color bar in all these figures shows $\mathrm{E}^{2}$. The bar range in all the figures is set between 0 and 2 for comparison. To compare the near-field effect, similar images for the standard cell are shown in Fig. S6. In the standard cell at a wavelength of about $400 \mathrm{~nm}$, the field intensity above the active layer is significant. Moreover, as the wavelength increases, the intensity increases further in the middle of the active layer.

The field intensity is the result of the interaction of light inside the cell structure with the NP shown on the cross section as well as other particles is present in the MNS but it does not exist on the selected surface. As can be seen, the intense near-field effect is more lateral and is in the vicinity of $\mathrm{NP}(\mathrm{s})$, especially when the nanoparticles are very close to each other (coupling NPs). By moving away from the MNS to the active layer $(\sim 10 \mathrm{~nm})$, the near-field effect decreases rapidly. But, in the opposite direction, the intensity of nearfield effects reduces slower. It seems that in O-S modes, the MNS does not lead to greater near-field intensity in the active layer. Here it appears that a slight change in MNS 

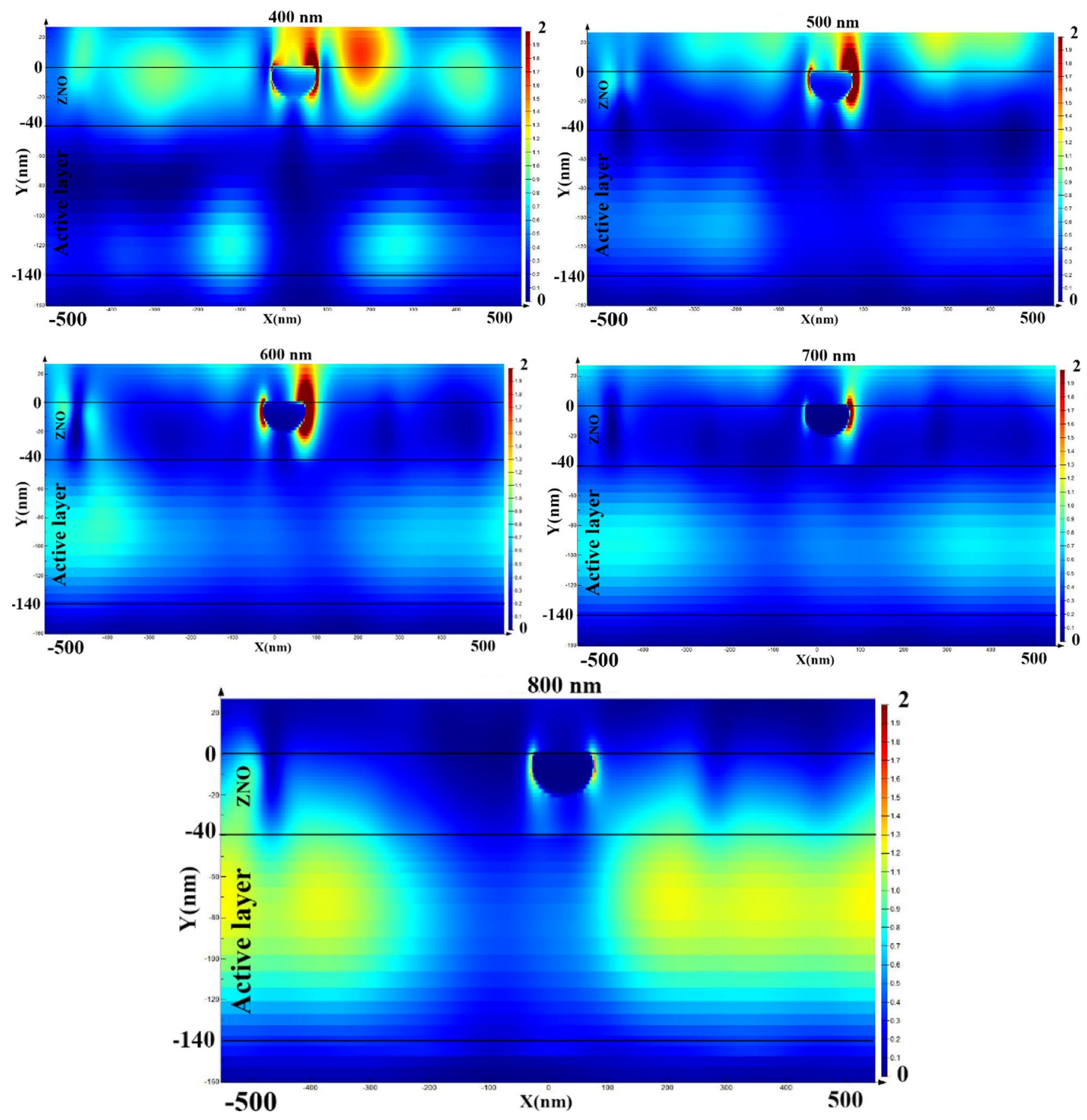

Fig. 7 Images of field intensity at different wavelengths $(400 \mathrm{~nm}, 500 \mathrm{~nm}, 600 \mathrm{~nm}, 700 \mathrm{~nm}$, and $800 \mathrm{~nm})$ on the selected cross section (Figs. 2 and 3 ) of the Al-O.2 mode (MNS at the interface of ITO/ZnO). The color bar in all the figures shows $E^{2}$

geometry can lead to a distinct change in near-field effect, in contrast to the far-field effects, which in some modes resulted in a bit of difference between the total reflection of the plasmonic sample and the standard. Differences can be shown by comparing O.2 and P.2 images (Figs. 7 and 8 ) and comparing both with the standard (Fig. S6). For the P.2 sample, intensity attenuation toward the active layer occurs more slowly in the active region of the solar cell. This can be attributed to the different geometry of the MNS and its lower surface coverage. Moreover, similar field intensity images for O.2 and P.2 (Ag and Au material) are given in Figs. S7-S10. It can be seen that by changing the material even in the same mode, the appearance of the near-field will vary. In the $\mathrm{O} .2$ mode (for $\mathrm{Al}$ and $\mathrm{Ag}$ ), the near-field effects for all five selected wavelengths are observed. But for Au, strong near-field effects are more seen at $700 \mathrm{~nm}$ and $800 \mathrm{~nm}$. 

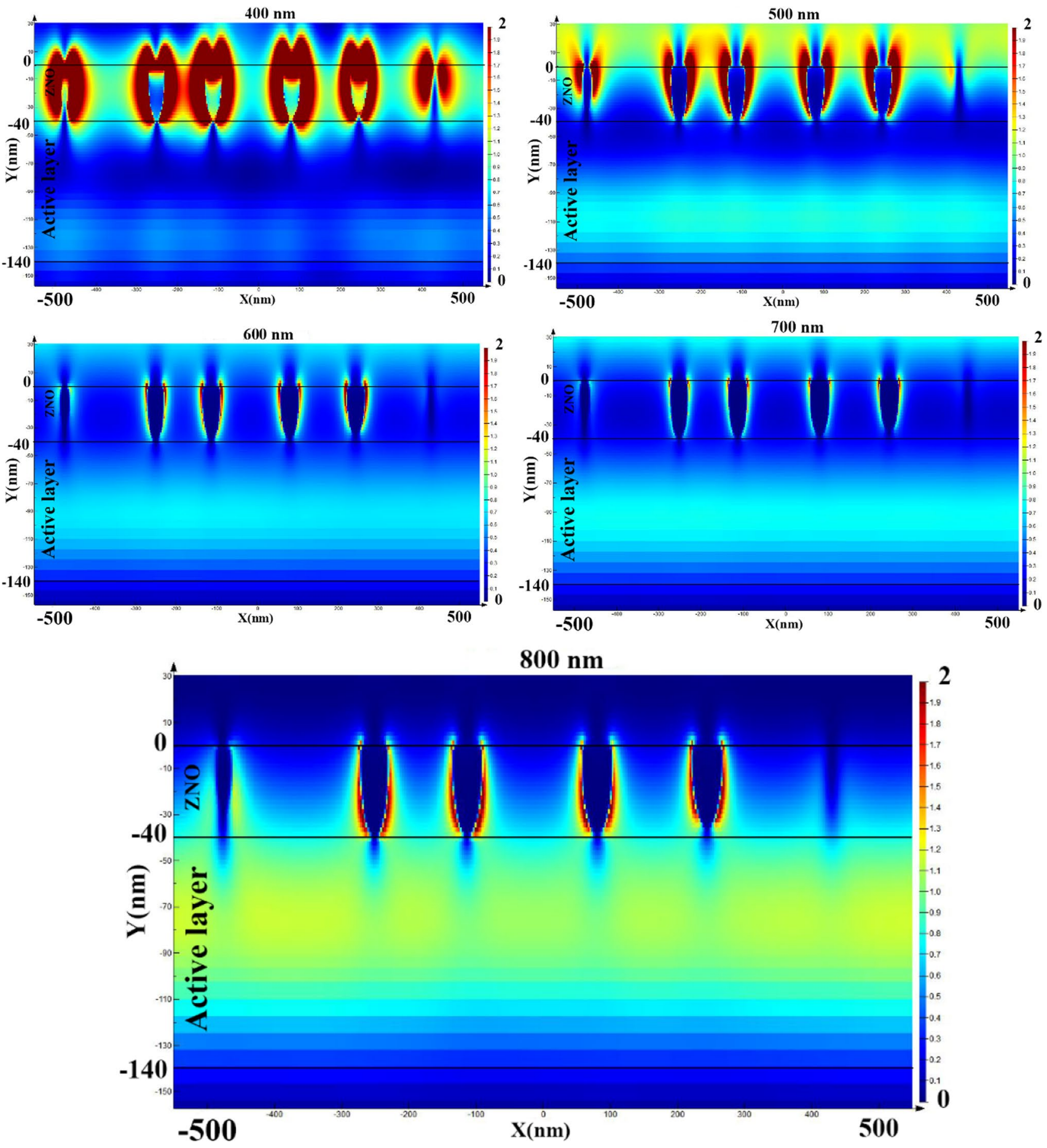

Fig. 8 Images of field intensity at different wavelengths $(400 \mathrm{~nm}, 500 \mathrm{~nm}, 600 \mathrm{~nm}, 700 \mathrm{~nm}$, and $800 \mathrm{~nm})$ on the selected cross section of the Al-P.2 mode (MNS at the interface of ITO/ZnO)

These results are somewhat in line with Fig. 6, where the maximum intensity wavelengths of $\mathrm{Au}$ are mostly seen at around $700 \mathrm{~nm}$ and $800 \mathrm{~nm}$. The same comparison in the P. 2 mode for three materials shows more exciting changes. In Fig. 7, in the five selected wavelengths, the field intensity inside the MNS does not have a significant value. But for the
P. 2 mode, especially at the image related to the wavelengths of $400 \mathrm{~nm}$ and $500 \mathrm{~nm}$ for $\mathrm{Ag}$ (Fig. S8) and wavelengths of 600 for $\mathrm{Au}$ (Fig. S10), there is a significant field intensity within the MNS. This powerful field intensity inside the structure can lead to parasitic loss absorption. Therefore, this behavior of the P. 2 mode (for Ag and Au compared to 
$\mathrm{Al}$ ) can be considered a negative effect on the application in the plasmonic SC design.

\subsection{Plasmonic NS (MNS) at the interface of the $\mathrm{ZnO} /$ active layer of organic solar cells}

Lastly, to more illustrate the possible differences, the MNS (P.2 mode) was added on the $\mathrm{ZnO} /$ active layer (i.e., directly in the active layer). In this case, the near-field images at selected wavelengths are given in Fig. 9 for Al (Fig. S11 and Fig. S12 for Ag and Au, respectively). A qualitative comparison of Figs. 7, 8, and 9 show that the Al-P.2 MNS at the $\mathrm{ZnO} /$ active layer is associated with changes in the form of the near-field compared to Al-P.2 MNS at ITO/ZnO. In other words, the intensity of the backward near-field at the wavelengths of $400 \mathrm{~nm}$ and $500 \mathrm{~nm}$ has been reduced to a reasonable extent, and the intensity of the forward near-field at the wavelengths of $600 \mathrm{~nm}$ and $700 \mathrm{~nm}$ has been increased. These can be useful in designing solar cell enhancement due to the near-field effect. The same comparison between Fig. S8 and Fig. S11 also shows remarkable differences. For Ag-P.2, the parasitic absorption at wavelengths of $400 \mathrm{~nm}$ and $500 \mathrm{~nm}$ is no longer observed when inserted at the $\mathrm{ZnO} /$ Active layer. In the case of $\mathrm{Au}$, the changes are somewhat reversed. On the other hand, in the Au-P.2 on $\mathrm{ZnO}$ /active layer, the parasitic absorption is intensified at $600 \mathrm{~nm}$ and $700 \mathrm{~nm}$ wavelengths compared to the ITO/ZnO case.

Generally, in terms of the near field, the Au causes an intense effect but it more appears in the form of parasitic absorption loss (Fig. S11 and Fig. S12). A slight parasitic absorption effect is seen to a small extent in the Ag sample, and its near-field amplifications are over a broader spectrum of wavelengths. In the $\mathrm{Al}$ case, it does not show parasitic absorption in the studied wavelength range.

The spectral reflection and the maximum field intensity of P.2 MNS ( $\mathrm{Al}, \mathrm{Ag}$, and $\mathrm{Au}$ ) on the interface of the $\mathrm{ZnO} /$ active layer are also shown in Fig. S13 and Fig. S14, respectively. Again, the reflectance difference is negligible compared to the standard sample.

\section{Conclusion}

In this study, an advanced FDTD model for MNS was presented. This model is based on some elliptical NPs whose average sizes and standard deviations are already determined. Some particles are placed almost randomly together to form the MNS. The NPs were divided into three groups $\mathrm{O}, \mathrm{P}$, and $\mathrm{S}$, and these groups were divided into several modes based on the height of NP. Considering the average specifications and their standard deviation, and the random rotation of the NPs relative to the vertical axis, different MNSs were prepared. This classification was made in such a way that it is close to some experimental structures, which could represent different contact angles and the forms of MNS close to some experimental methods. The results of the total reflectance of the modeled plasmonic samples and their comparison with the standard one as a criterion for investigating scattering (far-field effect) showed that none of them resulted in a significant reduction in reflectance across the whole spectrum. The maximum spectrum field intensity simulated on the selected cross sections was chosen as a criterion for investigating the near-field effect. These results were used to study the near-field effects and compare them qualitatively for various modes and materials. The study has shown that for MNS at the interface of ITO/ZnO, the peak distribution of $E^{2}{ }_{\text {max }}$ for $\mathrm{Al}$ and $\mathrm{Ag}$ versus $\mathrm{Au}$ is broader over the simulated spectrum. Instead, the numerical scale of $E^{2}{ }_{\max }$ for $\mathrm{Au}$ is higher. Then, the field intensity image in the visible spectrum and with a step of $100 \mathrm{~nm}$ was used to more compare the near-field effect. These images showed the extent of the near-field effect in different directions around the NPs and the field within the NPs themselves. These results illustrate that the amount of field distribution around the particle and the parasitic absorption is strongly dependent on the geometry of the MNS, wavelength, and its location. Al did not show much parasitic absorption in any mode and wavelength, unlike Au and Ag. Although the plasmonic effects of $\mathrm{Au}$ are more significant, the parasitic absorption loss of Au must be carefully considered at each mode. Lastly, the same studies were repeated when the MNS was placed at the interface of the $\mathrm{ZnO}$ /active layer. In fact, by adding MNS to the new location, changes are made in the near-field effects that are not the continuation of the previous ones (when MNS was at the interface of ITO/ZnO). In general, the Au modeling of the MNS samples shows the effects of severe parasitic absorption loss. Generally, the $\mathrm{Al}$ and relatively $\mathrm{Ag} \mathrm{MNS}$ at the interface of the $\mathrm{ZnO}$ /active layer can improve OSC performance due to optical reasons, especially by the near-field effect. Unlike Au and relative Ag, the Al sample did not show a parasitic absorption loss for MNSs. Therefore, the results of this study could be helpful to plasmonic designs.

\section{Supplementary material}

The more simulated outcomes for $\mathrm{Ag}$ and $\mathrm{Au}$, such as near and far-field results, are given in the supplementary material (information) for further consideration and comparison with the results of $\mathrm{Al}$. 

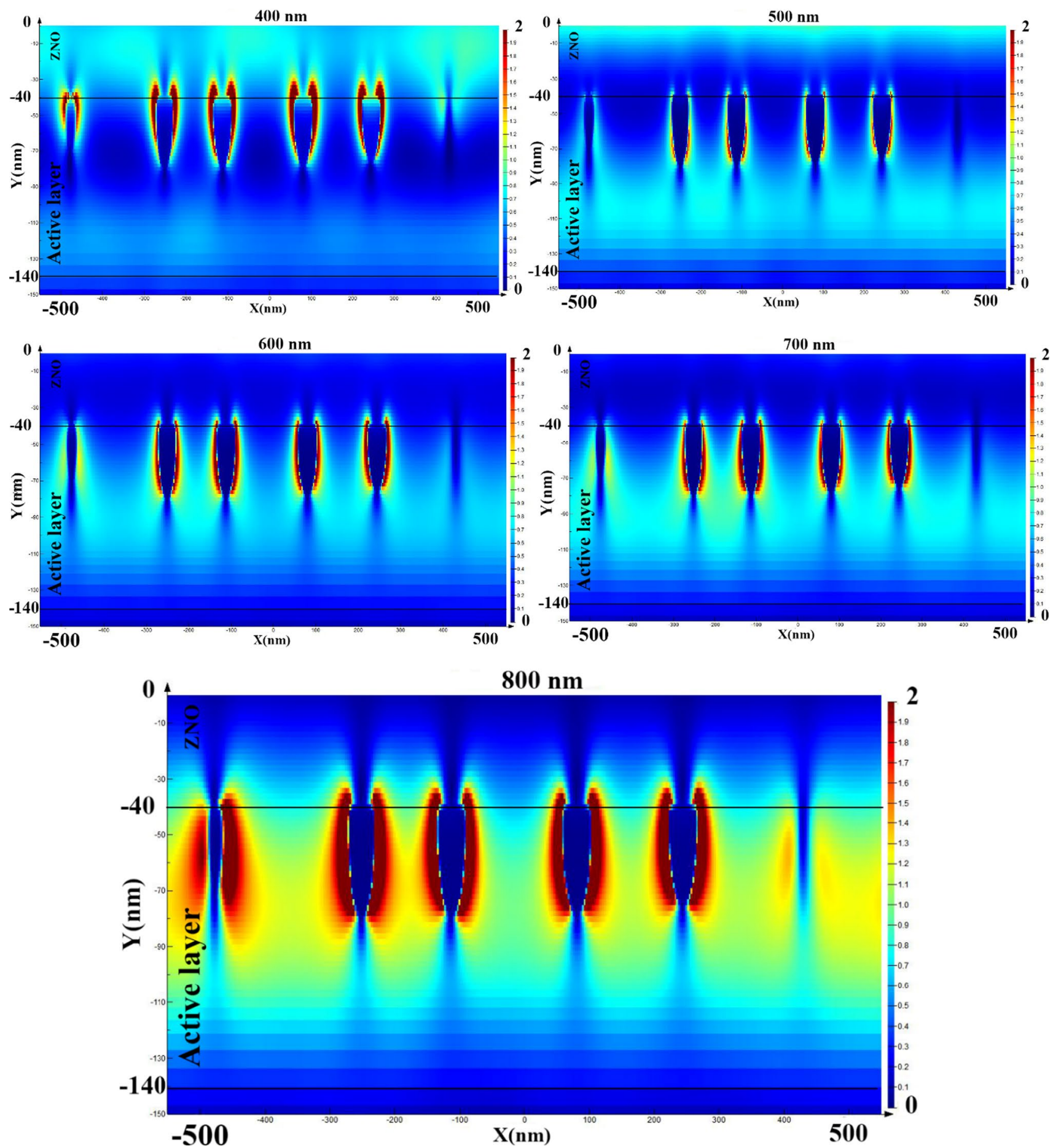

Fig. 9 Images of field intensity at different wavelengths $(400 \mathrm{~nm}, 500 \mathrm{~nm}, 600 \mathrm{~nm}, 700 \mathrm{~nm}$, and $800 \mathrm{~nm})$ on the selected cross section in which the Al-P.2 MNS was added to the interface of the $\mathrm{ZnO}$ /active layer

Supplementary Information The online version contains supplementary material available at https://doi.org/10.1007/s10825-021-01829-x.

Acknowledgements Thanks to Shahrekord University's rapid computing center for providing the simulation's hardware.
Author contributions Not applicable.

Funding Not applicable. 
Availability of data and material Due to the increased file size after each simulation (usually about $50 \mathrm{~GB}$ or more), their storage is faced with hardware limitations.

Code availability The Lumerical simulator is used for FDTD simulation.

\section{Declarations}

Conflict of interest The author declares no conflicts of interest.

Ethical approval Not applicable.

Consent to participate Not applicable.

Consent for publication Not applicable.

\section{References}

1. Newton, D.E.: World energy crisis: a reference handbook, ABCCLIO (2013)

2. Scales, A.J.: Civilization's Crisis: A Set of Linked Challenges. World Scientific Publishing Company, Singapore (2017)

3. Reinders, A., Verlinden, P., van Sark, W., Freundlich, A.: Photovoltaic Solar Energy: From Fundamentals to Applications. Wiley, New York (2017)

4. Voronov, M.: Organic Solar Cells: Advances in Research and Applications. Nova Science Publishers Incorporated, New York (2017)

5. Berny, S., Blouin, N., Distler, A., Egelhaaf, H.-J., Krompiec, M., Lohr, A., Lozman, O.R., Morse, G.E., Nanson, L., Pron, A., Sauermann, T., Seidler, N., Tierney, S., Tiwana, P., Wagner, M., Wilson, H.: Solar trees: first large-scale demonstration of fully solution coated, semitransparent, flexible organic photovoltaic modules. Adv. Sci. 3, 1500342 (2016)

6. Enrichi, F., Righini, G.C.: Solar Cells and Light Management: Materials, Strategies and Sustainability. Elsevier, New York (2019)

7. Stuart, H.R., Hall, D.G.: Island size effects in nanoparticleenhanced photodetectors. Appl. Phys. Lett. 73, 3815-3817 (1998)

8. Zhou, N., López-Puente, V., Wang, Q., Polavarapu, L., PastorizaSantos, I., Xu, Q.-H.: Plasmon-enhanced light harvesting: applications in enhanced photocatalysis, photodynamic therapy and photovoltaics. RSC Adv. 5, 29076-29097 (2015)

9. Jang, Y.H., Jang, Y.J., Kim, S., Quan, L.N., Chung, K., Kim, D.H.: Plasmonic solar cells: from rational design to mechanism overview. Chem. Rev. 116, 14982-15034 (2016)

10. Lim, E.L., Yap, C.C., Mat Teridi, M.A., The, C.H., Mohd Yusoff, A.R.B., Hj Jumali, M.H.: A review of recent plasmonic nanoparticles incorporated P3HT: PCBM organic thin film solar cells. Organ. Electron. 36, 12-28 (2016)

11. Ueno, K., Oshikiri, T., Sun, Q., Shi, X., Misawa, H.: Solid-state plasmonic solar cells. Chem. Rev. 118, 2955-2993 (2018)

12. Rau, U., Paetzold, U.W., Kirchartz, T.: Thermodynamics of light management in photovoltaic devices. Phys. Rev. B 90, 035211 (2014)

13. Yu, Z., Raman, A., Fan, S.: Fundamental limit of nanophotonic light trapping in solar cells. Proc. Natl. Acad. Sci. 107, 1749117496 (2010)

14. Yablonovitch, E.: Statistical ray optics. J. Opt. Soc. Am. 72, 899907 (1982)
15. Callahan, D.M., Munday, J.N., Atwater, H.A.: Solar cell light trapping beyond the ray optic limit. Nano Lett. 12, 214-218 (2012)

16. Kim, K., Lee, S.: Detailed balance analysis of plasmonic metamaterial perovskite solar cells. Opt. Express 27, A1241-A1260 (2019)

17. Huh, J.-H., Kim, K., Im, E., Lee, J., Cho, Y., Lee, S.: Exploiting colloidal metamaterials for achieving unnatural optical refractions. Adv. Mater. 32, 2001806 (2020)

18. Kim, K., Yoo, S., Huh, J.-H., Park, Q.H., Lee, S.: Limitations and opportunities for optical metafluids to achieve an unnatural refractive index. ACS Photonics 4, 2298-2311 (2017)

19. Lee, S.: Colloidal superlattices for unnaturally high-index metamaterials at broadband optical frequencies. Opt. Express 23, 28170-28181 (2015)

20. Pazos-Outón, L.M., Xiao, T.P., Yablonovitch, E.: Fundamental efficiency limit of lead iodide Perovskite solar cells. The Journal of Physical Chemistry Letters 9, 1703-1711 (2018)

21. Wu, B., Mathews, N., Sum, T.C.: Plasmonic Organic Solar Cells: Charge Generation and Recombination. Springer, Singapore (2016)

22. Fedlheim, D.L., Foss, C.A.: Metal Nanoparticles: Synthesis, Characterization, and Applications. Taylor \& Francis, London (2001)

23. Akimov, Y.A., Koh, W.S.: Resonant and nonresonant plasmonic nanoparticle enhancement for thin-film silicon solar cells. Nanotechnology 21, 235201 (2010)

24. Qi Xu, F.L., Liu, Y., Meng, W., Cui, K., Feng, X., Zhang, W., Huang, Y.: Aluminum plasmonic nanoparticles enhanced dye sensitized solar cells. Opt. Express 22, A301-A310 (2014)

25. Carretero-Palacios, S., Calvo, M.E., Míguez, H.: Absorption enhancement in organic-inorganic halide Perovskite films with embedded plasmonic gold nanoparticles. J. Phys. Chem. C 119, 18635-18640 (2015)

26. Carretero-Palacios, S., Jiménez-Solano, A., Míguez, H.: Plasmonic nanoparticles as light-harvesting enhancers in Perovskite solar cells: a user's guide. ACS Energy Lett. 1, 323-331 (2016)

27. Dusastre, V.: Materials for Sustainable Energy: A Collection of Peer-reviewed Research and Review Articles from Nature Publishing Group. World Scientific, Singapore (2011)

28. Schuster, C.S., Bozzola, A., Andreani, L.C., Krauss, T.F.: How to assess light trapping structures versus a Lambertian Scatterer for solar cells? Opt. Express 22, A542-A551 (2014)

29. Knight, M.W., King, N.S., Liu, L., Everitt, H.O., Nordlander, P., Halas, N.J.: Aluminum for plasmonics. ACS Nano 8, 834-840 (2014)

30. Gérard, D., Gray, S.K.: Special Issue on Aluminium Plasmonics. IOP Publishing, New York (2015)

31. Haber, J.A., Buhro, W.E.: Kinetic instability of nanocrystalline aluminum prepared by chemical synthesis; facile room-temperature grain growth. J. Am. Chem. Soc. 120, 10847-10855 (1998)

32. Martin, J., Plain, J.: Fabrication of aluminium nanostructures for plasmonics. J. Phys. D Appl. Phys. 48, 184002 (2014)

33. Lachebi, I., Fedala, A., Djenizian, T., Hadjersi, T., Kechouane, M.: Morphological and optical properties of aluminum nanoparticles deposited by thermal evaporation on heated substrates. Surf. Coat. Technol. 343, 160-165 (2018)

34. Fredriksson, H., Alaverdyan, Y., Dmitriev, A., Langhammer, C., Sutherland, D.S., Zäch, M., Kasemo, B.: Hole-mask colloidal lithography. Adv. Mater. 19, 4297-4302 (2007)

35. Inan, U.S., Marshall, R.A.: Numerical Electromagnetics: The FDTD Method. Cambridge University Press, Cambridge (2011)

36. Yu, W.: Advanced FDTD Methods: Parallelization, Acceleration, and Engineering Applications. Artech House, Norwood (2011)

37. Das, N.: Nanostructured Solar Cells. IntechOpen, London (2017)

38. Feng, L., Niu, M., Wen, Z., Hao, X.: Recent advances of plasmonic organic solar cells: photophysical investigations. Polymers 10, 123 (2018) 
39. Gaspar, D., Pimentel, A.C., Mateus, T., Leitão, J.P., Soares, J., Falcão, B.P., Araújo, A., Vicente, A., Filonovich, S.A., Águas, H., Martins, R., Ferreira, I.: Influence of the layer thickness in plasmonic gold nanoparticles produced by thermal evaporation. Sci. Rep. 3, 1469 (2013)

40. Parashar, P.K., Sharma, R.P., Komarala, V.K.: Plasmonic silicon solar cell comprised of aluminum nanoparticles: Effect of nanoparticles' self-limiting native oxide shell on optical and electrical properties. J. Appl. Phys. 120, 143104 (2016)

41. Rockett, A.: Thin Film Growth Processes. In: The Materials Science of Semiconductors, pp. 455-503. Springer, Boston, MA (2008). https://doi.org/10.1007/978-0-387-68650-9_10

42. Heilmann, A.: Polymer Films with Embedded Metal Nanoparticles. Springer, Berlin Heidelberg (2013)

43. Quan, J., Zhang, J., Qi, X., Li, J., Wang, N., Zhu, Y.: A study on the correlation between the dewetting temperature of $\mathrm{Ag}$ film and SERS intensity. Sci. Rep. 7, 14771 (2017)

44. Scholl, J.A., Koh, A.L., Dionne, J.A.: Quantum plasmon resonances of individual metallic nanoparticles. Nature 483, 421-427 (2012)

45. Malola, S., Lehtovaara, L., Enkovaara, J., Häkkinen, H.: Birth of the localized surface plasmon resonance in monolayer-protected gold nanoclusters. ACS Nano 7, 10263-10270 (2013)

46. Myroshnychenko, V., Rodríguez-Fernández, J., Pastoriza-Santos, I., Funston, A.M., Novo, C., Mulvaney, P., Liz-Marzán, L.M., García de Abajo, F.J.: Modelling the optical response of gold nanoparticles. Chem. Soc. Rev. 37, 1792-1805 (2008)

47. Mortensen, N.A., Raza, S., Wubs, M., Søndergaard, T., Bozhevolnyi, S.I.: A generalized non-local optical response theory for plasmonic nanostructures. Nat. Commun. 5, 3809 (2014)

48. McMahon, J.M., Gray, S.K., Schatz, G.C.: Nonlocal optical response of metal nanostructures with arbitrary shape. Phys. Rev. Lett. 103, 097403 (2009)

49. McMahon, J.M., Gray, S.K., Schatz, G.C.: Nonlocal dielectric effects in core-shell nanowires. J. Phys. Chem. C 114, 1590315908 (2010)
50. Toscano, G., Raza, S., Jauho, A.-P., Mortensen, N.A., Wubs, M.: Modified field enhancement and extinction by plasmonic nanowire dimers due to nonlocal response. Opt. Express 20, 4176-4188 (2012)

51. Fernández-Domínguez, A.I., Wiener, A., García-Vidal, F.J., Maier, S.A., Pendry, J.B.: Transformation-optics description of nonlocal effects in plasmonic nanostructures. Phys. Rev. Lett. 108, $106802(2012)$

52. Fernández-Domínguez, A.I., Zhang, P., Luo, Y., Maier, S.A., García-Vidal, F.J., Pendry, J.B.: Transformation-optics insight into nonlocal effects in separated nanowires. Phys. Rev. B 86, 241110 (2012)

53. Manzhos, S., Giorgi, G., Lüder, J., Ihara, M.: Modeling of plasmonic properties of nanostructures for next generation solar cells and beyond. Adv. Phys. X 6, 1908848 (2021)

54. Duan, H., Fernández-Domínguez, A.I., Bosman, M., Maier, S.A., Yang, J.K.W.: Nanoplasmonics: classical down to the nanometer scale. Nano Lett. 12, 1683-1689 (2012)

55. https://refractiveindex.info/.

56. FDTD: 3D Electromagnetic Simulator-Lumerical Inc. https:// www.lumerical.com

57. Gonçalves, M.R., Melikyan, A., Minassian, H., Makaryan, T., Marti, O.: Strong dipole-quadrupole coupling and Fano resonance in H-like metallic nanostructures. Opt. Express 22, 24516-24529 (2014)

58. Shopa, M., Kolwas, K., Derkachova, A., Derkachov, G.: Dipole and quadrupole surface plasmon resonance contributions in formation of near-field images of a gold nanosphere. Opto-Electron. Rev. 18, 421-428 (2010)

Publisher's Note Springer Nature remains neutral with regard to jurisdictional claims in published maps and institutional affiliations. 